\title{
Effect of chronic exposure to dexamethasone and its withdrawal on rocuronium-induced neuromuscular block
and sugammadex reversal in in vitro study of rats
}

(0)

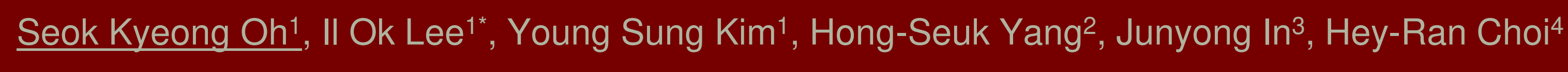

Department of Anesthesiology and Pain Medicine, ${ }^{1}$ Korea University Guro Hospital, ${ }^{2}$ Asan Medical Center, ${ }^{3}$ Dongguk University Ilsan Hospital, ${ }^{4}$ Seoul Paik Hospital, Seoul, South Korea

Background Chronic exposure to dexamethasone is associated with resistance to non-depolarizing neuromuscular blocking agents. Previous studies have reported that chronic dexamethasone treatment induced alteration and upregulation in immature subunits of nicotinic acetyl-choline receptor in diaphragm of rats. However there was no previous report about the effect of chronic exposure to dexamethasone on sugammadex (SGX) reversal in rat diaphragm.

Methods Male Sprague-Dawley rats (7-8 week-old; 202 to $271 \mathrm{~g}$ ) were randomized to receive a daily intraperitoneal injection of dexamethasone $(500 \mu \mathrm{g} / \mathrm{kg})$ in dexamethasone group or same amount of saline $(0.9 \% \mathrm{NaCl})$ in control for 14 days. At $1,3,7$ days after the last dexamethasone (group D1,D3,D7) (given the withdrawal time) or 1 day after saline (C), the rats were anesthetized. The hemi-diaphragm with attached phrenic nerve intact was immediately obtained.

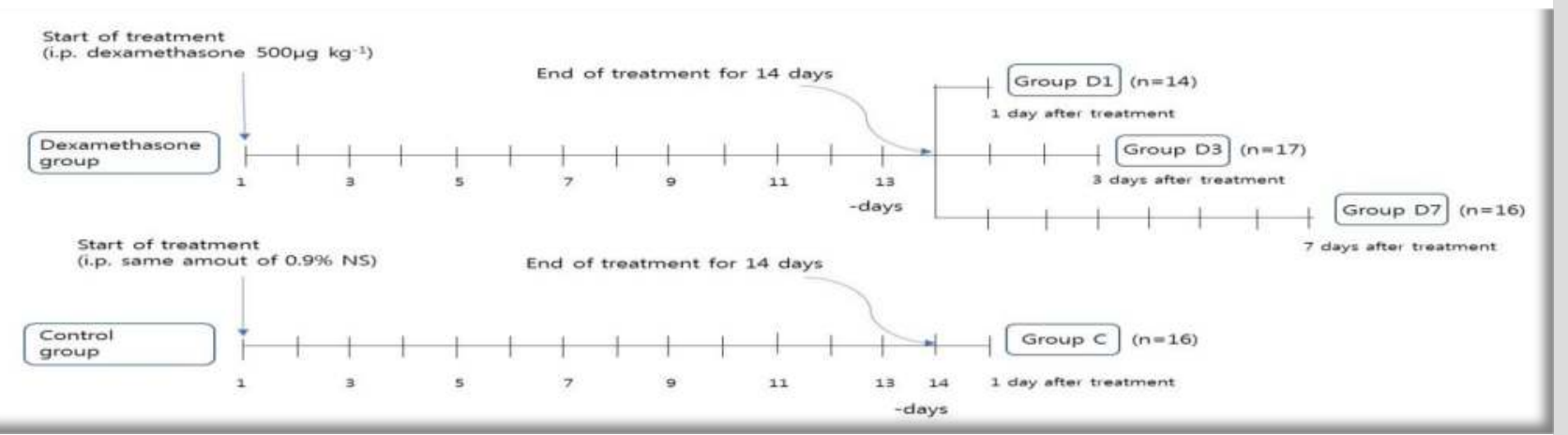

After the elicited twitch tension by phrenic nerve had been stabilized, Train-of-four (TOF) was determined. Rocuronium $250 \mu \mathrm{g}$ was then applied to the organ bath. Following stabilization, TOF was again determined with repetitive administration of cumulative dose of Rocuronium $250 \mu \mathrm{g}$.

After complete block ( $\mathrm{T} 1$ height $<5 \%$ ), SGX twice as much as rocuronium-equimolar dose was applied. Recovery profiles (time to TOFratio[TOFR] $>0.9$, recovery index [Dur25to75\%]) were observed. Expression of $\alpha 7-n A C h R$ subunit was assessed using immunohistochemistry. The correlation for the time to recovery and the other variables was conducted using Spearman's test.
Results There was no significant difference between groups in diaphragm size and weight.

Dose-response curve of rocuronium in group D1 was shifted to right compared to group $C(p<0.05)$, but curves in D3, D7 groups were not significantly different. IC50 in group D1 is significantly larger than group C. (Table 1)



Recovery time to TOFR $>0.9$ and recovery index were $12.8 \pm 4.9,15.0 \pm$ $7.1,10.2 \pm 3.3,10.1 \pm 3.8$ and $5.36 \pm 1.9,5.8 \pm 3.3,3.8 \pm 1.3,3.12 \pm 1.2$ (min) in group $C$ and group D1, D3, D7 respectively. It was not statistically significant in time to TOFR $>0.9$, but recovery index in group D7 were significantly shorter than group $C$ and D1. (Table 2)

The expression of $\alpha 7-n A C h R$ were detected on the membrane of skeletal muscle cell, which appeared on the all Dexa groups, peaked on the Dexa 1, and declined gradually on Dexa 3 and Dexa 7 group.

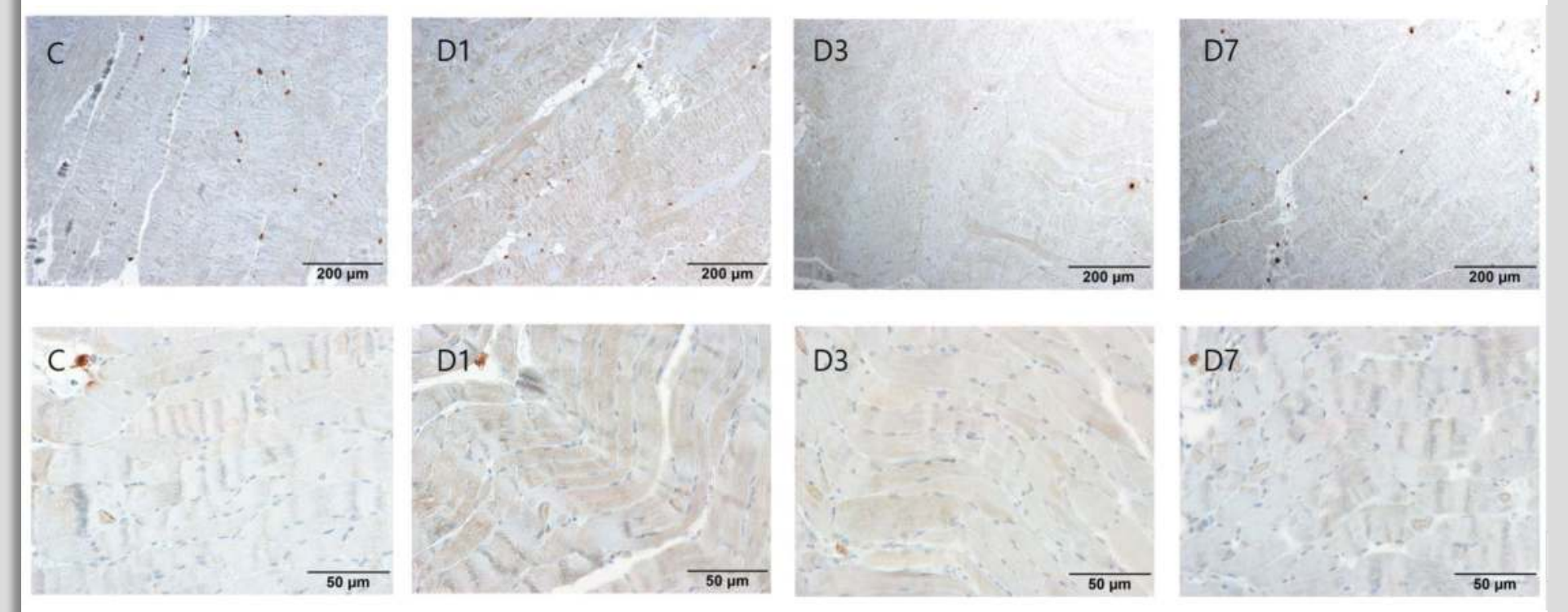
optical microscope (BX51T-32F01;: Olympus Corporation, Tokyo, Japan) upper row , $x 100$, scale bar $=200 \mu \mathrm{m}$; lower row , $x 400$, scale bar $=50 \mu \mathrm{m}$

Recovery profiles were positively correlated with IC50 value and amount of drugs. The time to recovery of NMB by SGX seemed to be correlated with the binding time of the NMBAs and SGX, not with the resistance property of receptors. (Table 3 )

Conclusions Chronic exposure to dexamethasone causes the resistance of the rat diaphragm to rocuronium. Furthermore, the pharmacodynamics change in dexamethasone treatment was recovered within 3 days after withdrawal (in 7-8 week-old rats). TOFR >0.9 when reversed with SGX was not affected by dexamethasone administration, however the recovery index in group D7 was shorter than group C and D1.

The chronic exposure to dexamethasone seemed not effect on sugammadex (SGX) reversal in rat diaphragm.

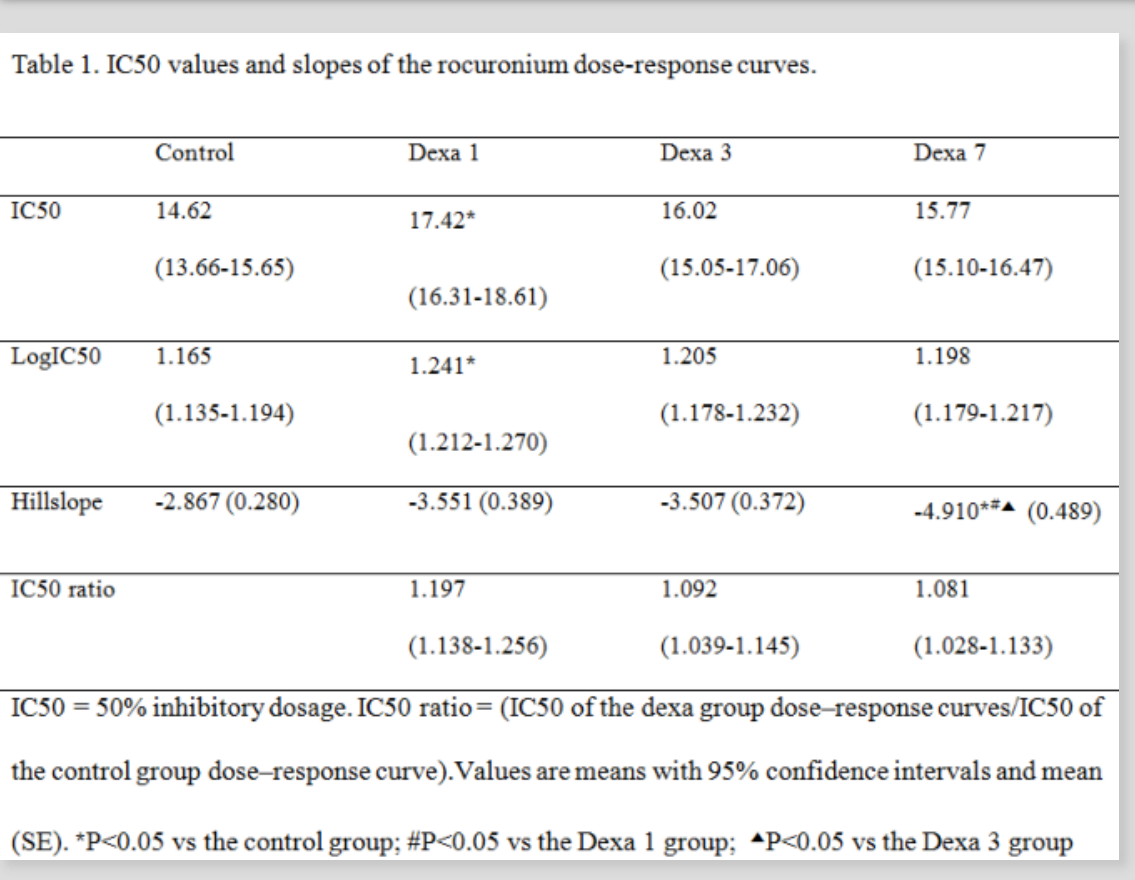

Reference Chen D, Yang MR, Huang LN, Qiu YW, Li ST. Genet Mol Res 2014; 13: 5892-900.

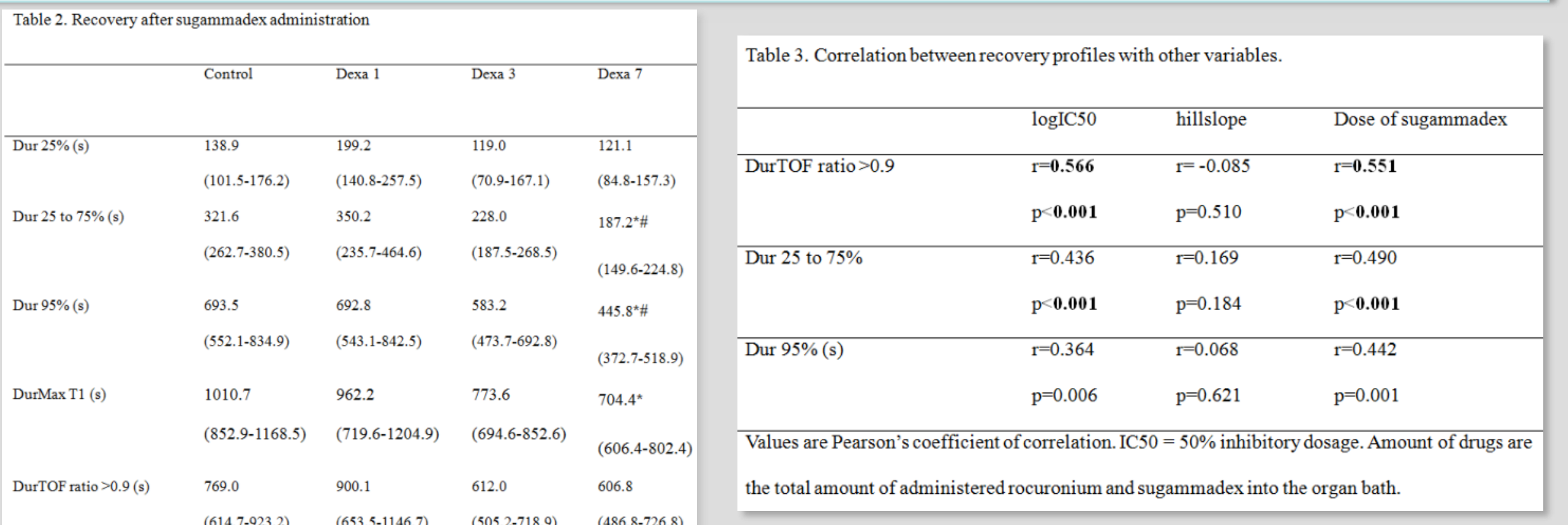

Contact Address : Korea Univ. Guro Hospital, Gurodong-ro 148, Guro-gu, Seoul 08308,,South Korea The Corresponding author (IO Lee) iloklee@korea.ac.kr The first author (SK Oh) nanprayboy@korea.ac.kr 